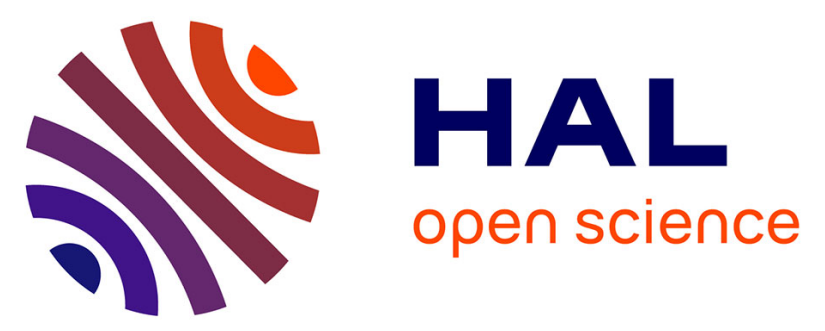

\title{
Rheology of polymer solutions using colloidal-probe atomic force microscopy
}

Ahmad Darwiche, François Ingremeau, Yacine Amarouchene, Abdelhamid Maali, Isabelle Dufour, Hamid Kellay

\section{To cite this version:}

Ahmad Darwiche, François Ingremeau, Yacine Amarouchene, Abdelhamid Maali, Isabelle Dufour, et al.. Rheology of polymer solutions using colloidal-probe atomic force microscopy. Physical Review E: Statistical, Nonlinear, and Soft Matter Physics, 2013, 87 (6), 062601 (10p.). 10.1103/PhysRevE.87.062601 . hal-00831670

\section{HAL Id: hal-00831670 \\ https://hal.science/hal-00831670}

Submitted on 13 Nov 2014

HAL is a multi-disciplinary open access archive for the deposit and dissemination of scientific research documents, whether they are published or not. The documents may come from teaching and research institutions in France or abroad, or from public or private research centers.
L'archive ouverte pluridisciplinaire HAL, est destinée au dépôt et à la diffusion de documents scientifiques de niveau recherche, publiés ou non, émanant des établissements d'enseignement et de recherche français ou étrangers, des laboratoires publics ou privés.

\section{다)(1) $(5$}

Distributed under a Creative Commons Attribution - NonCommerciall 4.0 International 


\title{
Rheology of polymer solutions using colloidal-probe atomic force microscopy
}

\author{
A. Darwiche, ${ }^{1,2, *}$ F. Ingremeau, ${ }^{1}$ Y. Amarouchene, ${ }^{1}$ A. Maali, ${ }^{1}$ I. Dufour, ${ }^{2}$ and H. Kellay ${ }^{1}$ \\ ${ }^{1}$ Université Bordeaux 1, LOMA UMR5798 CNRS, 351 cours de la Libération, 33405 Talence, France \\ ${ }^{2}$ Université Bordeaux 1, IMS UMR5218 CNRS, 351 cours de la Libération, 33405 Talence, France
}

(Received 20 February 2013; published 14 June 2013)

\begin{abstract}
We use colloidal-probe atomic force microscope (AFM) to study the rheological behavior of polymer solutions confined between two surfaces: the surface of a sphere and a flat surface on which the fluid is deposited. Measurements of the hydrodynamic force exerted on the sphere by the flowing liquid allowed retrieving the viscosity of the solution for different distances between the sphere and the flat surface. This method has been experimentally tested for Newtonian fluids for which the viscosity does not vary versus the gap dimensions. On the other hand, for non-Newtonian fluids, such as the large molecular weight polymer solutions used here, the measured viscosity depends on the gap height $D$ between the flat surface and the sphere. The decrease of the viscosity versus gap height is similar to previously observed variations in colloidal suspensions. Depletion of polymers in the gap region due to the high shear rates involved is a possible cause for such a variation.
\end{abstract}

DOI: 10.1103/PhysRevE.87.062601

PACS number(s): 83.80.Rs, 47.85.mf, 83.50.-v, 47.57.Ng

\section{INTRODUCTION}

Fluids such as polymer solutions, surfactant solutions, emulsions, or colloidal suspensions behave very differently from low molecular weight liquids such as water when a flow field is imposed. These differences are usually highlighted by the manner in which the viscosity of such solutions depends on the deformation rate and by the existence of a nonzero elastic modulus. This behavior is generally due to the presence of mesoscopic constituents that interact with each other and with the flow field. It is the complexity of these interactions that is responsible for the rich behavior of complex fluids. Different methods are used to characterize the behavior of these fluids subjected to a flow deformation. Rheological measurements turn out to be very sensitive to the nature of the fluid studied. The rheology of these fluids is a key issue in several domains of applications, such as food processing, materials processing, cosmetics, and lubrication, where the fluids are subjected to strong deformations. Conventional methods for measuring the rheological properties of a given fluid rely on the use of commercial rheometers using different geometries [1,2]. The classical rheometer [1,2] measures the viscosity of a fluid as a function of imposed shear stress or shear rate and provides information about the viscoelasticity (elastic and viscous moduli) of the probed fluid for different time scales. Despite the widespread use of this technique, it is limited by the performance of the device: a rheometer does not allow rheological measurements beyond a certain range of frequencies, for example, and demands large quantities of fluid for operation. To overcome the frequency-range limitation, alternative methods dubbed "microrheology" have been developed $[3,4]$. These methods are well adapted to miniaturization, thereby allowing the characterization of a given material using very small volumes. The ability to characterize small volumes of fluid enabled these techniques to be widely used in the field of biocharacterization, for example $[5,6]$. The major drawback of such a technique is the

\footnotetext{
"Present adress: Université Bordeaux 1, CBMN UMR 5248 CNRS, Allée de St Hilaire, 33600 Pessac, France.
}

long image and signal processing times needed to extract the rheological properties. Further developments also include the use of microelectromechanical systems (MEMS) to measure the local viscosity of fluids as well as their viscoelastic response in small volumes $[7,8]$. These usually rely on the use of miniature cantilevers that oscillate in the solution to be studied. The oscillations of the cantilever are sensitive to the ambient medium properties so quantities such as the viscosity of the solution or its elastic response can be extracted. The reduction in fluid volume accompanied by the reduction of the gaps used to create flow may pose a certain number of problems. When mesoscopic constituents are present (consider large molecular weight polymers in a liquid or colloids or nanoparticles in solution that introduce a characteristic size), if the gap used approaches a few characteristic sizes, the local concentration of the constituents may vary with solicitation. Recent calculations and observations have highlighted the fact that local concentrations of nanoparticles in small gaps may be much smaller than the bulk concentration, for example $[9,10]$. The role of confinement on the behavior of fluids is, therefore, essential not only for probing their properties at scales comparable to their constituents' dimensions but also for a better understanding of the results of different recent techniques.

In this paper, we use a colloidal-probe atomic force microscope to measure the rheological properties of confined polymer solutions between two surfaces: the surface of a sphere glued on the probe of a microcantilever and the flat surface holding the liquid. The principle of the technique is simple: if the sphere approaches the bottom surface at a constant velocity, the fluid exerts a hydrodynamic force on the sphere and this force gives direct information about the local viscosity of the fluid. This technique was used previously to study the hydrodynamic interaction between a fluid and a solid surface and to determine the boundary conditions for liquid flow near a solid surface [11-16].

The properties of the liquid near the solid surface play a major role in setting the forces acting on the sphere. In the 1960s, Brenner and others [17,18] have theoretically determined the hydrodynamic force on a colloidal particle flowing in a Newtonian fluid near a solid wall. This force 
depends on the separation distance between the sphere and the flat surface and on the viscosity of the fluid.

This hydrodynamic force has been tested explicitly by Honig and Ducker [19], who used colloidal-probe atomic force microscopy to study the hydrodynamic force exerted by the flow on a small sphere approaching a flat surface in a sucrose solution. Measurements of the viscosity of the solution, using the hydrodynamic force expression calculated by Brenner, turn out to be insensitive to the gap width as expected for a Newtonian fluid. It should be noted here that such a result may not be valid at gap widths comparable to the molecular dimensions of the fluid. Previous experiments [in surface force apparatus (SFA), for example, or using the colloidal probe] have observed ordering near solid surfaces for gap widths near five molecular sizes [20].

On the other hand, Bhattacharya and Blawzdziewicz [9] have carried out a theoretical analysis of the hydrodynamic force exerted on a colloidal particle approaching a solid surface in a dilute solution of smaller, nonadsorbing, spherical particles. The authors found that as the particle-flat surface gap width diminished, the viscosity in the gap region varied. At large separations with respect to the small particle diameter, the viscosity asymptotically approached that of the solution. At smaller distances, the viscosity is very close to the solvent viscosity. Specifically, the viscosity in the gap region was less than the solution viscosity at separation distances as large as 20 particle diameters. The local concentration of the small particles diminished as the gap between the sphere and the flat surface decreases. This calculation has been tested by G. K. James and J. Y. Walz [10], who used the colloidal-probe force microscopy technique to measure the hydrodynamic forces exerted on a 30- $\mu$ m-diameter sphere being moved toward or away from a flat surface in an aqueous solution of nanoparticles (22 $\mathrm{nm}$ diameter). They have shown, for large separation distances, that the measured viscosity of this confined solution is close to the viscosity measured using classical rheology. At small separation distances, they found, using the hydrodynamic force of Brenner, that the viscosity was smaller than that measured using classical rheology and close to that of the solvent. The viscosity measured did not depend on the velocity, and the changes in the effective viscosity extended to large (at least 20-nanoparticle diameters) separation distances. These results are consistent with the predictions of Bhattacharya and Blawzdziewicz [9].

Does such a phenomenology persist when the colloids are replaced by deformable objects such as polymers? Deformation of polymers in high shear rate regions or depletion of such molecules from such regions (as may occur near boundaries) has been observed before [21] and may affect the hydrodynamic resistance experienced by a sphere approaching a solid surface. In this paper, we examine this issue in detail.

In the experiments presented here, we have used the colloidal-probe atomic force microscopy technique to measure the force exerted on a sphere, as it approached a flat solid surface at different velocities, in a large molecular weight polymer solution. The separation distance between the sphere and the flat surface was varied from about $10 \mu \mathrm{m}$ down to a fraction of a micrometer. These separations span a range of distances from about 50 molecular diameters down to less than
1 molecular diameter. Confinement effects were, therefore, probed over a wide range of distances with respect to the polymer molecular dimensions. The diameter of the (probe) sphere was much larger than the molecular dimensions and was in the range of 115 to $125 \mu \mathrm{m}$. Through the hydrodynamic force exerted on the sphere, we deduce the viscosity of the fluid in the gap as a function of the velocity, the distance $D$ separating the sphere from the flat surface, and the shear rate $\dot{\gamma}$ within this gap. We systematically compared the measurements made by colloidal-probe atomic force microscopy to measurements using classical rheology. Our main results indicate that the measured viscosity is systematically smaller than the viscosity measured using a classical rheometer when the separation distance decreases. The effect is found even at distances as large as 20 times the radius of gyration of the polymer used. Depletion of polymer molecules in the gap region, most probably due to the high shear rates in the gap [21], is a probable reason for such a decrease of the viscosity of the solution. Complementary measurements using the Brownian motion of small particles in the gap region confirm these observations.

\section{MATERIALS AND METHODS}

On a silicon nitride rectangular cantilever (Veeco ORC8) of $200 \mu \mathrm{m}$ in length and $40 \mu \mathrm{m}$ in width, we glued a borosilicate sphere of diameter ranging from 115 to $125 \mu \mathrm{m}$ (GL0186B/106-MO-Sci Corporation) using an epoxy glue. The advantage of using such large-sized spheres is to increase the hydrodynamic interaction and to minimize the contribution of the hydrodynamic drag force due to the cantilever itself. A small droplet of the studied liquid was deposited on a hydrophilic mica surface. The cantilever and the sphere were immersed in the droplet. Before each experiment, the mica substrate was cleaved, ensuring a clean and smooth surface. The sample (mica surface) was fixed on a piezoelectric stage (Nano-T225, City Labs Inc., Madison, WI, USA), which has a displacement of $5 \mu \mathrm{m} / \mathrm{V}$.

The displacement of the piezoelectric system is controlled by a function generator using triangular waveforms with frequencies in the range of $0.01-1 \mathrm{~Hz}$ and a peak-to-peak amplitude of $8 \mathrm{~V}$ to move the fluid sample toward the probe and away from it. During the displacement of the sample, the deflection of the cantilever due to the hydrodynamic force exerted by the displacement of the fluid on the solid surface toward the probe is detected using a photodetector. An acquisition system is used to acquire the electrical signal from the photodetector.

We studied different fluids in this configuration: water- $\mathrm{NaCl}$ at $10 \mathrm{mM}$, an aqueous solution of polyethylene glycol [PEG; from Fluka Chemika Cat. No. 81300 Lot No. 408095/1; $\mathrm{M}_{w}=20000 \mathrm{~g} / \mathrm{mol}(16000-24000 \mathrm{~g} / \mathrm{mol})$, radius of gyration $R_{g}=4 \mathrm{~nm}$, and polymer overlap concentration $c^{*}=124000$ $\mathrm{ppm}$; the Zimm relaxation time is $\tau_{\text {zimm }}=1.6 \cdot 10^{-8} \mathrm{~s}$, the concentration used is $18300 \mathrm{ppm}$ or $\left.c / c^{*}=0.15\right]$ and an aqueous solution of a large molecular weight polymer polyacrylamide (PAA; Polyscience Cat. No. 18522, Lot No. $518644 ; \mathrm{M}_{w}=18 \times 10^{6} \mathrm{~g} / \mathrm{mol}, R_{g}=300 \mathrm{~nm}$ ) at different concentrations. The polymer overlap concentration $c^{*}$ for PAA 
is about $200 \mathrm{ppm}$ [22]. The estimated Zimm relaxation time of this polymer is $6.8 \mathrm{~ms}$. We examined concentrations both below and above $c^{*}$ for the PAA solution. Rheological measurements were performed in a controlled stress rheometer (AR1000, TA Instruments) in cone-plate geometry (radius $30 \mathrm{~mm}$, cone angle $1^{\circ}$ ). The truncated gap (the smallest distance between the cone and the plate) is $15 \mu \mathrm{m}$. This is larger than the range of gaps explored using the AFM.

\section{A. Hydrodynamic force}

Our experiments make explicit use of the hydrodynamic force exerted on a sphere as it approaches a flat solid surface. This problem has been solved in the 1960s by Brenner, and we here recall the main result. For a solid sphere approaching a flat solid surface with a velocity $V$, the hydrodynamic force acting on the sphere is given by Brenner's expression [17]:

$$
F=-6 \pi \eta R V \lambda,
$$

where

$$
\begin{aligned}
\lambda= & \frac{4}{3} \sinh (\alpha) \sum_{n=1}^{\infty} \frac{n(n+1)}{(2 n-1)(2 n+3)} \\
& \times\left\{\frac{2 \sinh [(2 n+1) \alpha]+(2 n+1) \sinh (2 \alpha)}{4 \sinh ^{2}\left[\left(n+\frac{1}{2}\right) \alpha\right]-(2 n+1)^{2} \sinh ^{2}(\alpha)}-1\right\}
\end{aligned}
$$

and

$$
\alpha=\cosh ^{-1}\left(1+\frac{D}{R}\right) .
$$

In Eqs. (1)-(3), $R$ is the sphere radius, $D$ is the separation distance between the surface of the sphere and the mica surface, and $\eta$ is the liquid viscosity.

In the case where $D$ is very small compared to the sphere radius $(D \ll R), \lambda=\frac{R}{D}$ and the force expression reduces to Taylor's form:

$$
F=-\frac{6 \pi \eta R^{2}}{D} V .
$$

On the other hand, when the distance $D$ is very large compared to the sphere radius $(D \gg R)$, the force expression simply reduces to the Stokes's form:

$$
F=-6 \pi \eta R V
$$

These expressions assume no slip boundary conditions on both the sphere surface and the flat surface.

\section{B. Cantilever calibration}

The measurements carried out here are based on recording the deflection of the cantilever with the sphere attached as the bottom flat surface is approached toward the sphere or retracted from it. The hydrodynamic force exerted by the fluid on the sphere causes the cantilever deflection. In order to extract the fluid characteristics, the cantilever properties and contact position with the flat surface need to be determined. The calibration of the cantilever deflection was performed in situ by making use of the cantilever deflection measured by the photodiodes as the mica substrate approached the sphere in a solution of water [23]. The addition of salt to the water reduces electrostatic forces between the mica surface and the sphere.

To convert the photodiode voltage to deflection in nanometers and get the contact position $(D=0)$ we have followed the procedure described in Refs. [19,24]. For each realization, we determine the slope of the measured photodiode voltage versus the piezo displacement in the constant compliance zone when the sphere and the substrate are in contact. This gives the cantilever deflection $z_{c}$. Extrapolation of the constant compliance zone data to zero intercepts the displacement axis at the contact position $(D=0)$, allowing us to determine the latter with a precision of roughly $5 \mathrm{~nm}$. This procedure is explained in Fig. 1(a). In order to obtain the actual separation distance, the cantilever deflection was added to the piezo displacement. The actual velocity (during the approach or withdrawal phases) is obtained from the full sphere-substrate distance by calculating its time derivative:

$$
V=\frac{d D}{d t} \text {. }
$$

A typical example of the measurement of the cantilever deflection resulting from the hydrodynamic force acting on the sphere is presented in Fig. 1(b). The deflection of the cantilever is positive when the mica surface approaches the sphere. On the other hand, when the mica surface is withdrawn from the sphere surface, the deflection is negative. The approach phase (A) and the withdrawal phase are similar in shape down to about 400-nm separation as shown in the inset to Fig. 1(b). This inset displays the sum of the deflections [upon approach (A) and upon withdrawal (W)], which is zero down to $400 \mathrm{~nm}$, showing that the withdrawal and approach phases are symmetric for distances larger than $400 \mathrm{~nm}$. The difference between the deflections is also shown and deviations due to an asymmetry between the two phases also appears at distances smaller than $400 \mathrm{~nm}$. This asymmetry between the approach and withdrawal is due to differences in the approach and withdrawal velocities at these distances.

The hydrodynamic force is related to the measured cantilever deflection by $F_{h}=k z_{c}$. From Eq. (4), which is valid for $D \ll R$, we have

$$
z_{c}=\frac{6 \pi \eta R^{2}}{k D} \frac{d D}{d t} .
$$

To determine the stiffness $k$ of the cantilever with a sphere attached, we plot $\frac{\left(\frac{d D}{d t}\right)}{z_{c}}$ versus $D$. This variation is linear with a slope given by $\frac{k}{6 \pi \eta R^{2}}$, as shown in Fig. 1(c). The stiffness is obtained from the value of the slope, for a known viscosity and known radius, giving $k=0.20 \pm 0.01 \mathrm{~N} / \mathrm{m}$.

\section{VISCOSITY MEASUREMENTS}

For the salt water solution, the only force that acts on the sphere during the approach or withdrawal of the solid surface is the viscous hydrodynamic force. For a polymer solution there could be additional forces (depletion forces, elastic forces, etc.) that may result from the confinement and the shearing of the solution. We note that while the viscous force changes direction if the velocity changes direction, the additional forces do not. To extract the effective viscosity of the studied liquids from the measured force, we proceed as follows. 

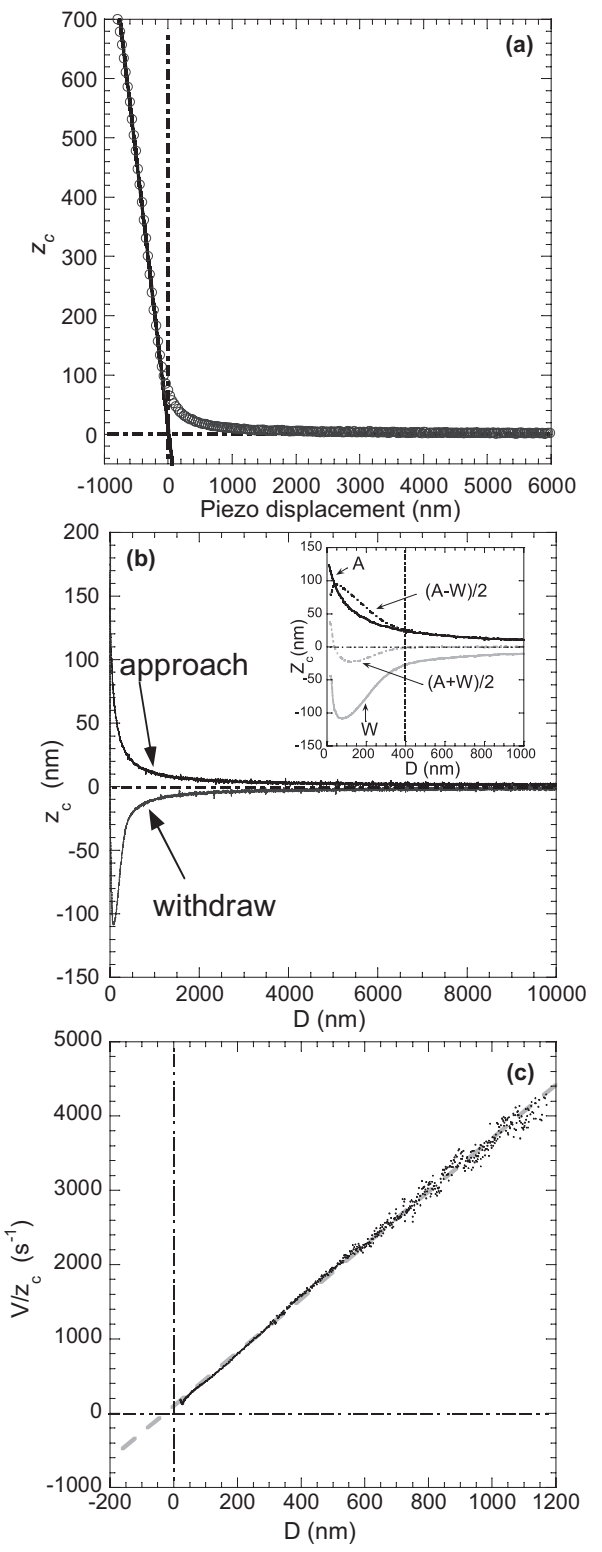

FIG. 1. (a) Cantilever calibration. Note the linear region giving the calibration of the photodetector voltage. Once this calibration is carried out and the voltage converted to a distance $z_{c}$, extrapolation of this linear zone to zero (indicated by the solid line) gives the contact position $D=0$. (b) Cantilever deflection due to the hydrodynamic force versus the distance $D$ between the surfaces (mica and sphere) during the displacement of the mica surface (surface carrying the liquid). For the approach, the deflection is positive and increases as $D$ decreases. For the withdrawal phase, the deflection is negative. The inset shows a close up at small distances $D$. Note that the approach and withdrawal signals are not symmetric for distances below $400 \mathrm{~nm}$, as shown by the nonzero value of the sum of deflections and by the deviation of the half difference between the deflections measured upon approach and upon withdrawal. This asymmetry at small distances is due to differences in velocity $V$ between the approach and the withdrawal phases at similar gap heights. (c) Variation of the velocity $V$ divided by the cantilever displacement versus the distance $D$. The confined liquid between the borosilicate sphere and the flat mica surface is salt water. The solid line is the fitting curve using the theoretical expression of Eq. (7). The fit goes through the origin, indicating no slip, and gives the value of the stiffness $k$.
We consider that the force acting on the sphere has two components, a viscous force $F_{v}$ and an additional force $F_{e}$. When the mica surface moves toward the sphere glued on the cantilever, the projection of the force gives

$$
F_{v}+F_{e}=k Z_{a} .
$$

On the other hand, when the mica surface moves away from the sphere, the projection of the force gives

$$
-F_{v}+F_{e}=k Z_{r} .
$$

Here, $Z_{a}$ and $Z_{r}$ are the cantilever deflections during the approach and withdrawal phases. While the viscous force changes sign, the additional forces do not. The sum and subtraction of Eqs. (12) and (13) gives

$$
\begin{aligned}
& 2 F_{e}=k\left(Z_{a}+Z_{r}\right), \\
& 2 F_{v}=k\left(Z_{a}-Z_{r}\right) .
\end{aligned}
$$

From the viscous force, and using the hydrodynamic force discussed above, we can extract the effective viscosity of the solution:

$$
\eta=\frac{k\left(Z_{a}-Z_{r}\right)}{12 \pi R V \lambda}
$$

\section{RESULTS AND DISCUSSION}

As explained above, the measurement of the deflection of the cantilever as a function of the distance $D$ allows us to obtain the viscous hydrodynamic force from an analysis of the approach and withdrawal phases. We have used two polymer solutions: a PEG solution of low molecular weight and exhibiting little elasticity and a PAA solution of high molecular weight with an important elastic response. In Figs. 2(a) and 2(b), we show the measured deflection versus the distance between the sphere and the flat surface for the PEG and the PAA solutions, respectively. In the case of the PEG solution [Fig. 2(a)], we observe that the deflection of the cantilever is symmetric between the approach and withdrawal phases. Any asymmetry arises at distances smaller than $400 \mathrm{~nm}$ as seen in Fig. 1(b). As expected, the only forces acting on the sphere are of viscous origin and no sign of an elastic response or additional forces is observed. However, these deflections are not symmetric, even at large distances, in the case of PAA solutions as shown in Fig. 2(b). The difference between both cases is related mostly to the presence of a strong elastic component for PAA. We cannot rule out the presence of additional forces, however. In order to unravel the information conveyed by the variation of the cantilever deflection versus distance $D$, we represent, as shown in Fig. 3(a), the sum and the difference of the deflections for PEG solutions using two different velocities. The sum of deflections is close to zero for the PEG solution as expected. In this case, the force exerted on the sphere surface is solely the viscous force, which is low for large separations and increases markedly for low separations and for higher velocities as expected. In Fig. 3(b), we plot the sum and the difference of the deflections measured for the PAA solution. We find that the deflections' sum is not zero but positive and increases as the separation distance decreases. This confirms the fact that the polyacrylamide solution is a viscoelastic liquid with a strong elastic component 

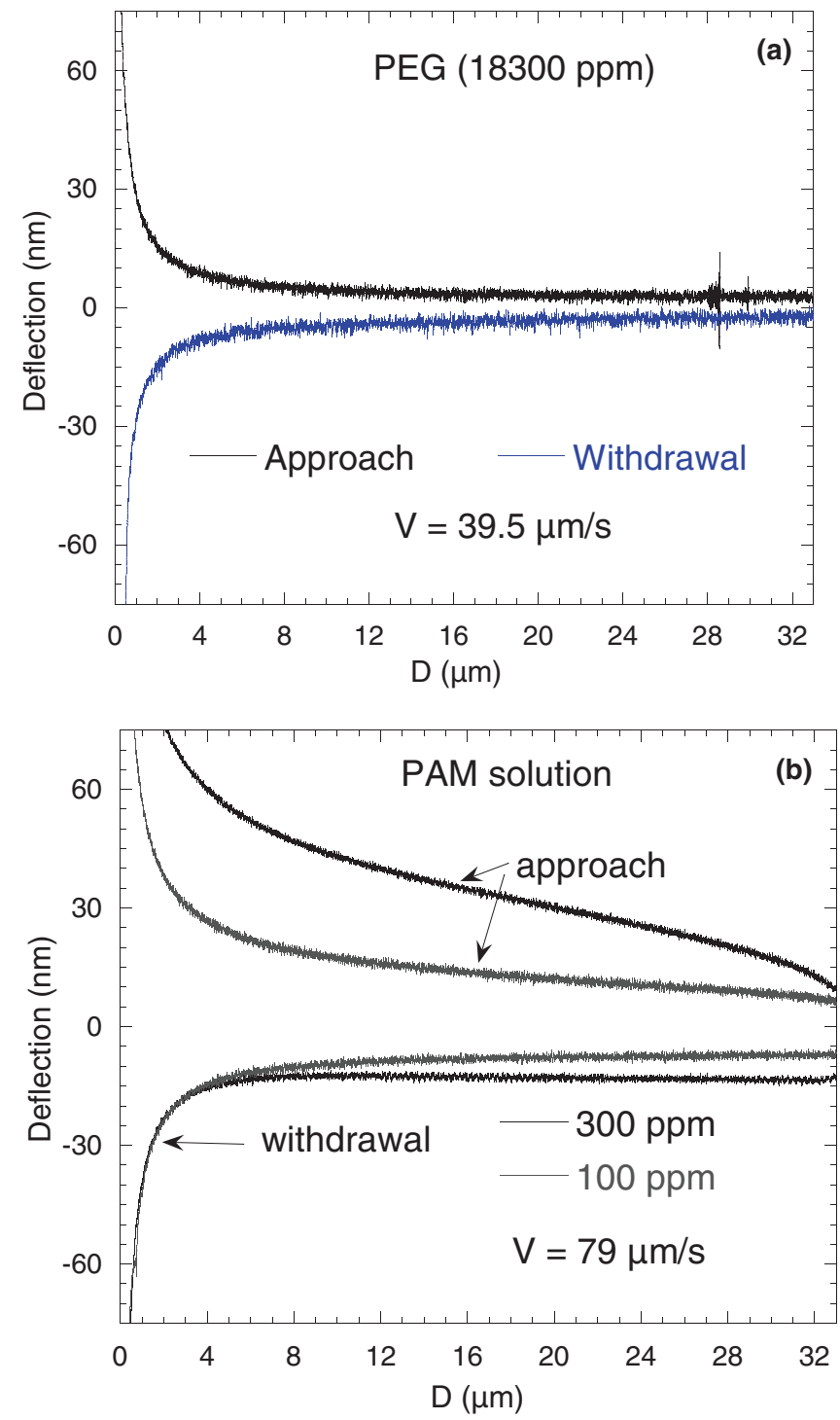

FIG. 2. (Color online) Measurement of the cantilever deflection during the approach and withdrawal of the mica surface. (a) In the case of Newtonian PEG solutions, symmetrical deflections between the approach and withdrawal of the cantilever were observed. (b) In the case of non-Newtonian polyacrylamide solutions, asymmetrical cantilever deflections have been observed. Two different concentrations (300 and $100 \mathrm{ppm}$, corresponding to 1 and 0.5 for $c / c^{*}$ ) are used for a velocity of $79 \mu \mathrm{m} / \mathrm{s}$. This velocity refers to that of the piezoelectric stage used to move the mica surface and not to the relative velocity between the mica surface and the sphere.

giving rise to a positive normal force. Possible depletion forces, which are on the other hand attractive, cannot be ruled out even though their magnitude was estimated to be much smaller than the measured forces at the examined separations. The forces exerted on the surface of the sphere in this case are both elastic and viscous forces, which increase as the separation distance decreases and as the polymer concentration increases.

Equation (12) can be used to calculate the viscosity of the polymer solutions versus the gap height $D$. Specifically, for PEG, which has a constant viscosity over a large range of shear rates as measured with a Rheometer and for which the elastic part is basically absent, we find that the viscosity
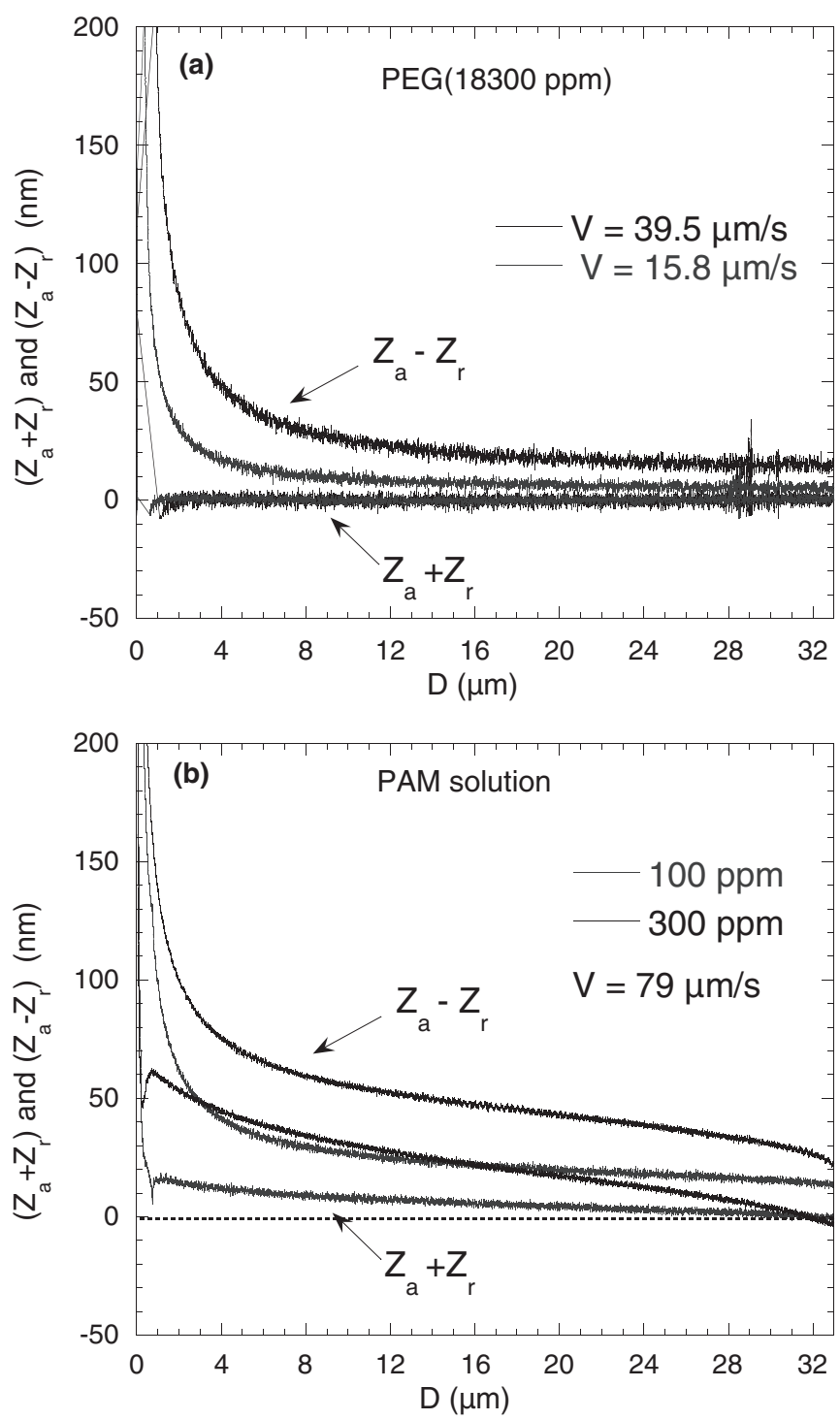

FIG. 3. Sum and difference of deflections: (a) Newtonian liquid is PEG at $18300 \mathrm{ppm}$ at different velocities of the mica surface and (b) non-Newtonian liquid PAA solution at 300 and 100 ppm for a velocity of $79 \mu \mathrm{m} / \mathrm{s}$. This velocity refers to that of the piezoelectric stage and not to the relative velocity between the surface and the sphere.

curves obtained during the approach and the withdrawal phases are superimposed. The value of the viscosity of the PEG solution shown in Fig. 4(a) turns out to be constant and independent of the distance $D$. These results are consistent with the rheology measurements for which the viscosity is independent of shear rate giving $\eta=0.007 \mathrm{~Pa} \mathrm{~s}$, as shown in Fig. 4(b). Note that the molecular size of the PEG molecules is well below the heights used.

As mentioned above, the PAA solution has elastic (and perhaps additional forces) and viscous contributions to the measured force. We will focus only on the viscous part obtained using Eq. (12). Since these solutions are strongly shear-thinning, we first consider the variation of the viscosity versus shear rate and estimate the shear rate as

$$
\dot{\gamma} \sim 3 \frac{V}{D} \text {. }
$$



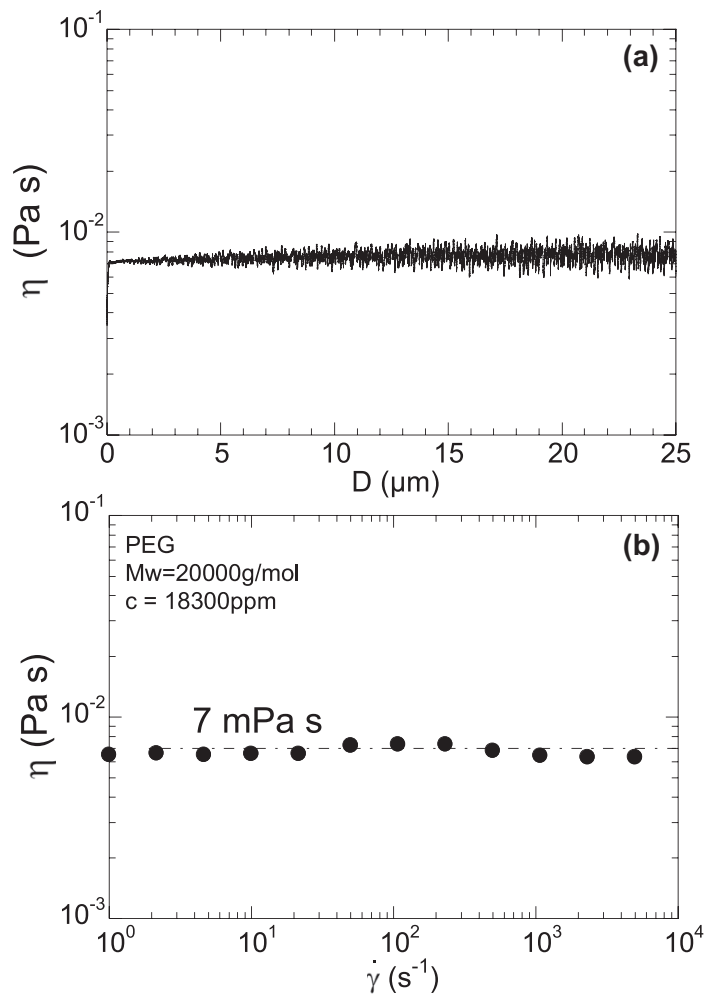

FIG. 4. (a) Viscosity of the PEG solution versus gap height $D$. (b) Viscosity of the PEG solution using rheology measurements in a cone plate geometry. Note that the two determinations give a similar result: a constant viscosity of about $7 \mathrm{mPa}$.

This estimate considers a volume $\pi D^{3}$ around the center of the gap and assumes Poiseuille flow. In Fig. 5, we plot the viscosity of the polyacrylamide solution at a concentration of $200 \mathrm{ppm}$ (parts per million by weight) or a value of $c / c^{*}$ of 1 extracted from the force measurements versus the estimated shear rate. The black curve represents the measurements obtained with a conventional method (a rheometer in the cone plate geometry) and the three other curves are obtained with the colloidal-probe AFM at different velocities of the piezoelectric stage.

From the rheology measurements, the viscosity is roughly constant at low shear rates and decreases with shear for rates higher than about $50 \mathrm{~s}^{-1}$, which corresponds to the inverse of the relaxation time of the solution. The viscosity measured with the AFM probe does not follow this variation and the measurements seem to be below the rheology curve and reach values close to the solvent viscosity for high shear rates. The two viscosities seem to be close only at low shear rates or large distances $D$. In order to explore this discrepancy with respect to conventional measurements even further, we compare data using different velocities of the piezoelectric stage. In principle, and if the viscosity depends only on the shear rate, the results obtained with the three different velocities used should superimpose. It is clear from Fig. 5 that the three viscosity curves deduced from the measurement of the cantilever deflection are offset relative to each other. The different realizations show that the viscosity of a polyacrylamide solution decreases when the shear rate increases but they do not collapse versus shear

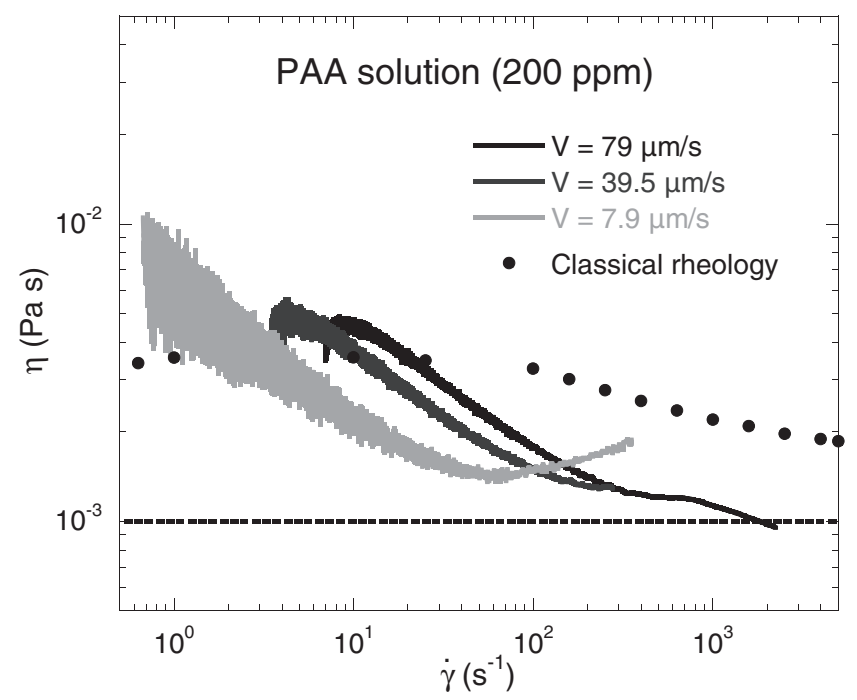

FIG. 5. Viscosity of the PAA solution $\left(200 \mathrm{ppm}, c / c^{*}=1\right)$ as a function of shear rate using different velocities. The results from AFM measurements are compared to rheological measurements. Note the differences between the measurements for different velocities and with the rheological measurements. The velocity refers to that of the piezoelectric stage and not to the relative velocity between the surface and the sphere.

rate as they should. All three curves are also offset from the rheology measurements. This behavior shows that the decrease of the viscosity observed here is not solely due to the shear thinning character of the solution. If this were the case, the viscosity curves should collapse versus shear rate for the three different velocities used and should agree with the rheology measurements.

On the other hand, if the viscosity is examined versus the distance $D$ between sphere and surface, a surprising result emerges. Figure 6(a) shows that the curves of the viscosity at a concentration of $200 \mathrm{ppm}$ for different piezoelectric stage velocities are superimposed when plotted versus the distance $D$. This shows that the viscosity is independent of the displacement velocity of the mica surface. This is reminiscent of the results mentioned above on nanoparticle suspensions, where the effective viscosity of the solution decreases with decreasing gap height due to a lowering of the suspension concentration in the gap region $[9,10]$. Note that for large gaps the viscosity is close to that of the bulk solution at low shear rates but the decrease persists at distances as high as $6 \mu \mathrm{m}$ (about 10 molecular diameters). Our results also explore heights comparable to and even smaller than the molecular dimensions of the polymers. For such small dimensions where the gap can hardly accommodate more than one molecule, it is probably not surprising to find small viscosities.

Other concentrations show a similar behavior. The decrease in viscosity when the distance $D$ between the two surfaces decreases is obtained for a $100 \mathrm{ppm}$ solution or a value of $c / c^{*}$ of 0.5 as shown in Fig. 6(b) for different displacement velocities. A summary of these results is shown in Fig. 7. The measured viscosity depends on the concentration of polymer and on the distance between the surface and the sphere but is independent of the velocity. As the concentration increases, the viscosity increases as expected. For large distances, the 

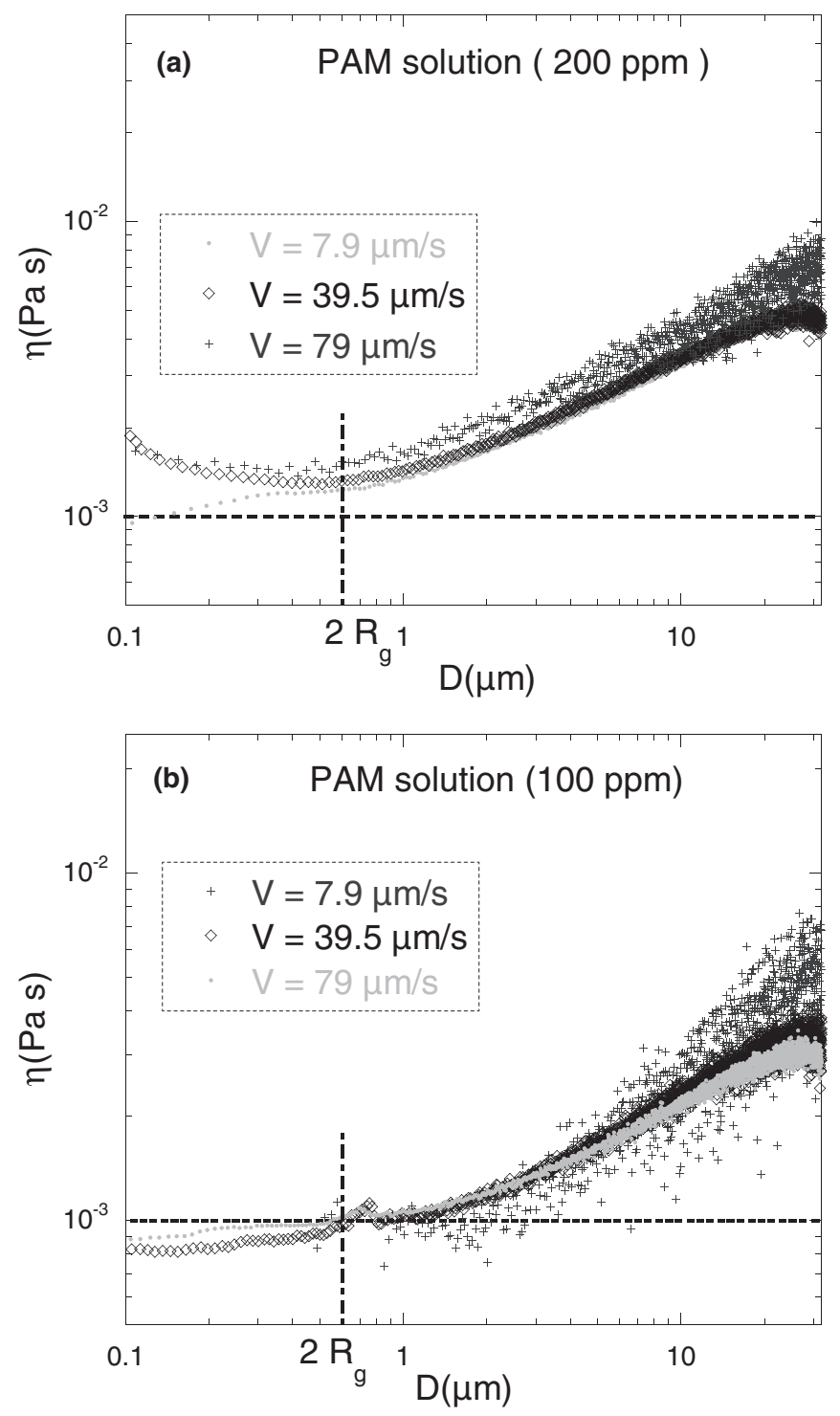

FIG. 6. (a) Viscosity of a PAA (200 ppm) solution at different velocities $(79 \mu \mathrm{m} / \mathrm{s}, 39.5 \mu \mathrm{m} / \mathrm{s}$, and $7.9 \mu \mathrm{m} / \mathrm{s})$ versus the distance $D$. (b) Viscosity of a PAA $\left(100 \mathrm{ppm}, c / c^{*}=0.5\right)$ solution at different velocities $(79 \mu \mathrm{m} / \mathrm{s}, 39.5 \mu \mathrm{m} / \mathrm{s}$, and $7.9 \mu \mathrm{m} / \mathrm{s})$ versus the distance $D$. Note that the different curves collapse onto a single curve. Note also that the large distance measurements are close to the low shear rate viscosity of the solution, which is near $4 \mathrm{mPa}$ s for the $200 \mathrm{ppm}$ solution and near $2 \mathrm{mPa}$ s for the $100 \mathrm{ppm}$ solution. The velocity refers to that of the piezoelectric stage and not to the relative velocity between the surface and the sphere.

extracted viscosity follows the viscosity measured with the rheometer. The inset to this figure shows the low shear rate viscosity from rheology measurements and that extracted at large distances. The two viscosities follow the same trend with values close to each other for the different concentrations examined. As the distance decreases, however, the viscosity shows a large decrease and reaches values close to the solvent viscosity for small distances.

The distance $D$ is, therefore, an important parameter that controls the viscosity variation. The shear rate as estimated from the simple expression above does not seem to be

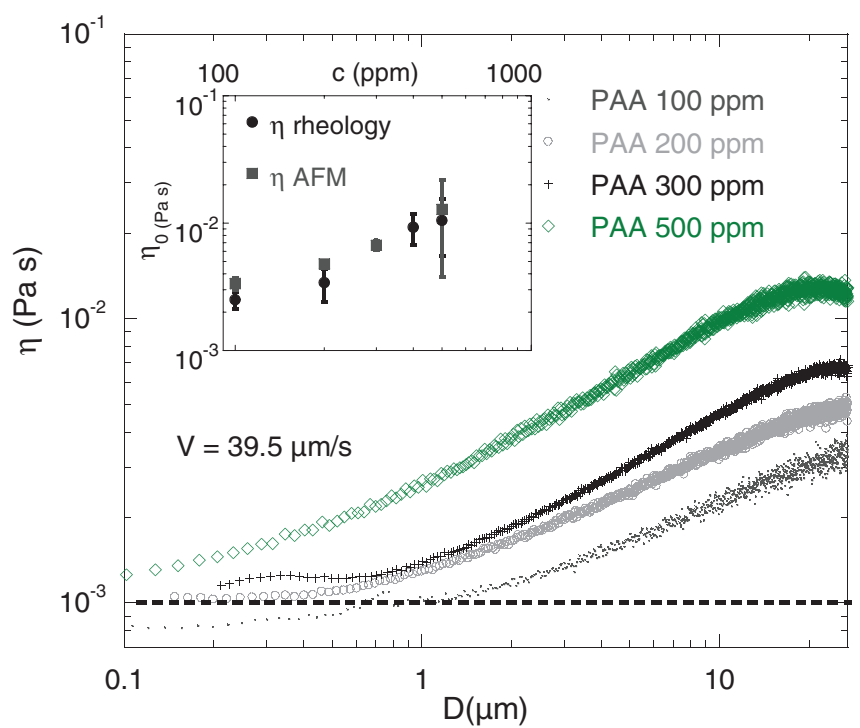

FIG. 7. (Color online) Viscosity of the PAA solution at different concentrations $\left(500,300,200\right.$, and $100 \mathrm{ppm}$, corresponding to $\mathrm{c} / \mathrm{c}^{*}$ values of $2.5,1.5,1$, and 0.5 ) for a velocity of $39.5 \mu \mathrm{m} / \mathrm{s}$. This velocity refers to that of the piezoelectric stage and not to the relative velocity between the surface and the sphere. The inset shows the zero shear viscosity from rheology measurements and the AFM measurements at large separation $D$.

a relevant parameter to collapse the viscosity data. Shear thinning alone is, therefore, not sufficient to explain the variation of the viscosity measured here. Furthermore, the fact that the viscosity decreases to values as small as that of the solvent cannot be explained by shear thinning alone since the rheology measurements indicate that at high shear rates (above $1000 \mathrm{~s}^{-1}$ ) the viscosity remains higher than that of the solvent.

One possible explanation for such a decrease of the viscosity for small distances $D$ (below about $5 \mu \mathrm{m}$ ) is depletion of polymers from the gap. This has been observed and predicted for colloidal solutions where the lowering of the particle concentration in the gap region is responsible for a lower viscosity. This effect has been noted for gap heights as large as 20 particle diameters. For the case of polymer solutions, it has been noted before that large shear rates induce migration of polymers away from the solid boundaries where the shear is high [21]. Such a depletion layer has been observed experimentally in different experiments [21] and its thickness $L$ is predicted to scale as $L \approx R_{g} \mathrm{Wi}^{2 / 3}$. Here, $\mathrm{Wi}$ is the so called Weissenberg number given by $\mathrm{Wi}=\dot{\gamma} \tau_{r}$, where $\tau_{r}$ is the relaxation time of the polymer solution. Typical depletion layer thickness is, therefore, comparable or higher than the radius of gyration $R_{g}$ of the molecules for Wi greater than 1 . The radius of gyration of the PAA molecules used here is $0.3 \mu \mathrm{m}$. We may, therefore, expect that at small distances and, therefore, relatively high shear rates, both near the surface of the sphere as well as on the flat surface, a depletion layer almost devoid of polymers exists that renders the hydrodynamic force acting on the sphere smaller than expected. This effect will give rise to a viscosity that is smaller than that at the macroscopic scale measured with the Rheometer. Note that for the PEG solution, the radius of gyration is estimated at $4 \mathrm{~nm}$ [25], and the relaxation time of the solution is estimated to be smaller 
than $0.1 \mathrm{~ms}$, so depletion effects may arise only at very small distances of order a few nanometers, so it is not surprising that the measured viscosity in this case remains close to that of the solution for gap heights larger than $80 \mathrm{~nm}$.

Further checks of such a hypothesis follow through measurements of the Brownian motion of small colloidal particles in the gap between the sphere and the surface. These additional experiments explore the Brownian motion of small particles in the gap between the sphere and the surface and, therefore, probe the local viscosity of the solution within the gap. If depletion does occur, we may expect enhanced diffusion with respect to the bulk polymer case and, therefore, a lower effective viscosity.

Fluorescent nanoparticles of diameter $200 \mathrm{~nm}$ were used in the solution. The concentration of particles is kept extremely small so no particular effects due to the presence of particles in solution are expected. The same large sphere diameter was used for this experiment, but instead of mica as the bottom surface, a microscope glass slide was used. The concentration of polymer (PAA) was fixed to $500 \mathrm{ppm}(c / c *=2.5)$. The sphere was approached toward the surface at a velocity of $180 \mu \mathrm{m} / \mathrm{s}$ down to a distance $D$ of $4 \mu \mathrm{m}$ from the surface. The movement was then stopped and the particles tracked using an epi-fluorescence set up with a microscope objective of $63 \times$ magnification. A sensitive camera working at 120 frames per second was used to image the particles and their Brownian motion in the gap between the sphere and glass slide.

The mean-square displacement of the nanoparticles was calculated from a time trace of the particles' trajectories as $1 / 2\left\langle[x(t+\tau)-x(t)]^{2}+[y(t+\tau)-y(t)]^{2}\right\rangle$. The brackets refer to an average over time $t$ and over different particles. Different positions from the center of the gap were examined and different times after cessation of the flow were analyzed.

Figure 8 displays the mean-square displacement of these nanoparticles in the bulk polymer solution, below the sphere in the polymer solution, and in the reference salt water solution. Note that the mean-square displacement varies linearly with time $\tau$ as expected for Brownian motion in all three cases. The slope of this variation gives the diffusion constant $D_{\mathrm{SE}}$, which is given by $\frac{k_{B} T}{6 \pi \eta R_{p}}$, where $k_{B}$ is the Boltzmann constant, $T$ is the temperature, $\eta$ is the fluid viscosity, and $R_{p}$ is the nanoparticle radius. The slope extracted from the water case gives a value of the viscosity of $1.3 \pm 0.4 \mathrm{mPa}$ s slightly higher but close to that expected for water. Similarly, the slope for the polymer solution gives a value of $7.5 \pm 3 \mathrm{mPa} \mathrm{s}$, which is slightly lower than the viscosity of the solution measured at low shear rates (we expect $10 \mathrm{mPa}$ s). The value of the slope for the polymer solution confined between the sphere and the surface right after cessation of the flow is, however, higher than that of the solution itself. This value corresponds to a viscosity of $3 \pm 1 \mathrm{mPa} s$ and is, therefore, smaller than the value of the viscosity of the polymer solution measured in the rheology experiments or in the Brownian diffusion experiments. This result is consistent with the fact that the AFM probe measurements suggest a smaller value for the viscosity of the solution at a similar gap height. For a gap height of $4 \mu \mathrm{m}$, the probe experiments give a value of $5 \mathrm{mPa}$, while that from the Brownian motion gives a value of $3 \mathrm{mPa}$. Both values are smaller than those measured for much larger gaps.

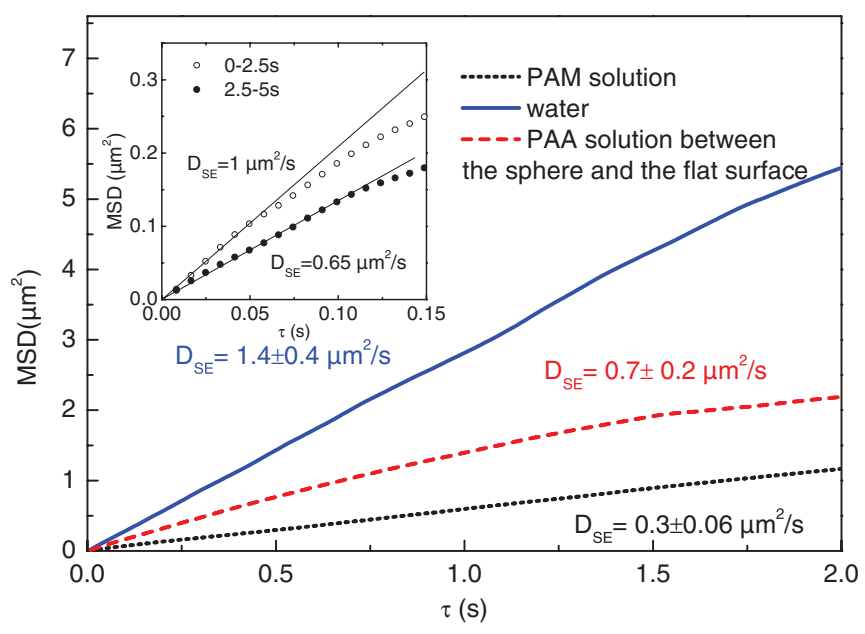

FIG. 8. (Color online) Mean-square displacement (in one dimension) of 200-nm-diameter spheres in bulk water, bulk polymer solution, and for a confined polymer solution between a sphere and a flat surface. The sphere's displacement was at a velocity of $180 \mu \mathrm{m} / \mathrm{s}$. The measurements started after the sphere's displacement was stopped at a distance of $4 \mu \mathrm{m}$ from the flat surface. The measured diffusion constant is given. The inset shows the meansquare displacement of one particle, in the gap between the sphere and the surface for two different time periods. Note that for later times after cessation of the sphere's movement toward the flat surface, the diffusion constant decreases, indicating a higher viscosity.

A further examination of these results was carried out by studying the mean-square displacement at different times after cessation of the shear, i.e., after the sphere was stopped. These results are shown in the inset of Fig. 8. The diffusion constant was found to decrease slowly with time, indicating that the observed depletion at early times slowly disappears after the cessation of the flow as the polymer solution relaxes to its equilibrium concentration in the gap region.

The inset of the upper graph of Fig. 9 shows a typical velocity profile versus the distance to the center $r$ (the points are the velocities measured using particle tracking of $200 \mathrm{~nm}$ particles at $3 \mu \mathrm{m}$ from the flat plate for $D=4 \mu \mathrm{m}$ ). The line is the expected velocity profile assuming Poiseuille flow in the gap in the same conditions as the measurements. The reasonable agreement between the measurements and the expected variation allows us to estimate, in this configuration, the shear rate at the wall. Another estimate of the shear rate in the gap can also be obtained by dividing the velocity of the tracked particles by the distance to the wall; this estimate is necessarily lower than the calculated shear rate at the wall using the assumption of a Poiseuille velocity profile in the gap as indirectly suggested by the inset of the upper graph of Fig. 9. These shear values are then multiplied by the relaxation time of the solution $(0.1 \mathrm{~s})$ [26] to obtain the Weissenberg number, which is plotted in the upper graph of Fig. 9. The difference between the data points obtained by dividing the velocity by the distance to the wall to obtain the shear rate (solid squares) and the solid line that uses Poiseuille flow in the gap clearly indicates that dividing the velocity of the particles by the distance to the wall underestimates the shear rate but may also indicate deviations from Poiseuille flow, which are difficult to observe due to measurement errors. In 


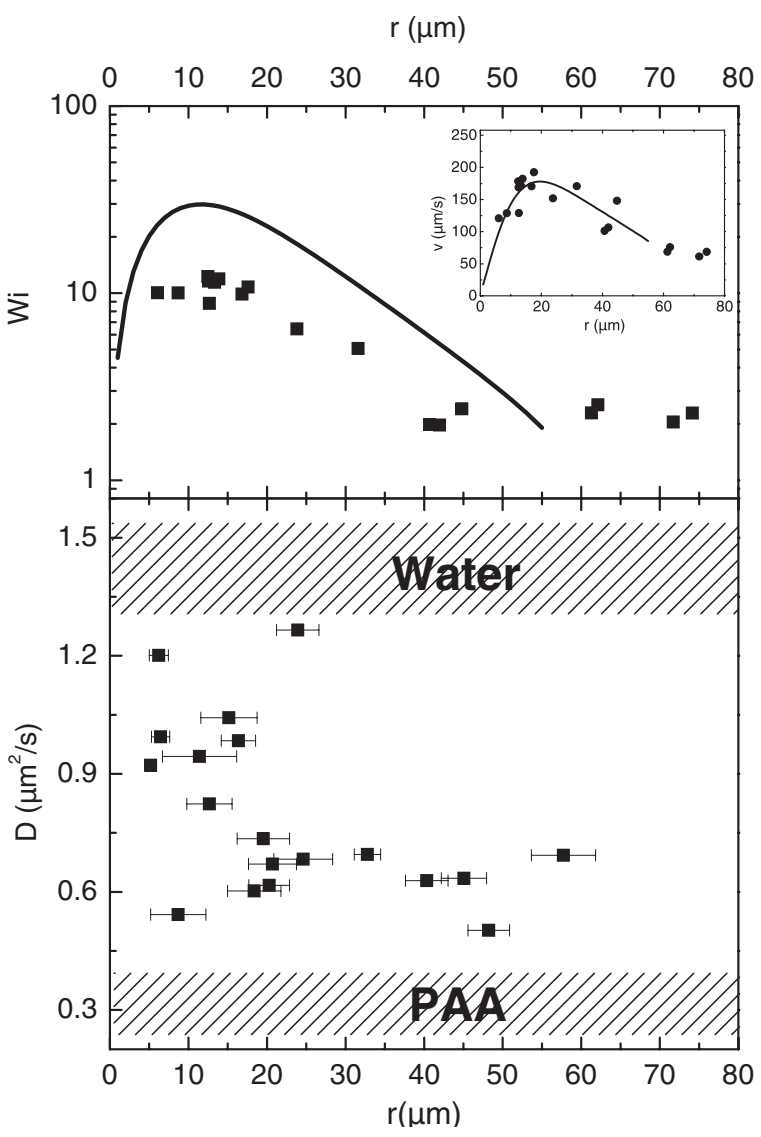

FIG. 9. The lower graph displays the diffusion coefficient for the small particles (obtained by tracking several particles) in the gap region for different distances from the center of the gap. Note that near the center, these diffusion constants are high, indicating smaller viscosities for the polymer solution. As the distance to the center increases, the diffusion constant decreases, indicating higher viscosities. The inset in the upper graph shows the variation of the velocity versus distance $r$ from the center of the gap along with the expected profile assuming Poiseuille flow. These measurements and the calculated profile are for $D=4 \mu \mathrm{m}, V=180 \mu \mathrm{m} / \mathrm{s}$, and at $3 \mu \mathrm{m}$ from the flat surface. The main graph shows the estimated Weissenberg number versus distance from center using the expected profile as well as the measured velocities. Note that high Weissenberg numbers are obtained for distances where the viscosity is low, indicating the importance of polymer migration and depletion.

addition to this, we have measured the diffusion constant of the nanoparticles at different distances $r$ from the center of the gap. The result is shown in the bottom panel of Fig. 9. Note that farther away from the center, the diffusion constant is small, albeit still slightly higher than the bulk value. When the distance to the center decreases and, therefore, the gap height decreases, the diffusion constant increases, indicating a lower viscosity and, therefore, a smaller mean concentration of polymers. The shear rates are high where the diffusion constants are significantly higher than those measured for large gaps. These shear rates give Weissenberg numbers of the order of 10 in a large area extending up to $20 \mu \mathrm{m}$ from the center of the gap (see Fig. 9, upper panel). These results suggest depletion layers of thickness greater than the molecular diameter. These measurements point to the importance of shear in the observed deviations from expected behavior based on rheology measurements of the viscosity and to the importance of shear-induced migration of polymers.

Note that it is not confinement alone that gives the trend in diffusion constant [27]: confinement generally reduces the diffusion constant, while here it increases as the gap height decreases. These results, therefore, comfort our AFM measurements in the sense that the approach of the sphere toward the flat surface may induce depletion of polymers within the gap, causing the viscous force to be smaller than expected and giving Brownian probes a higher diffusion coefficient.

\section{CONCLUSIONS}

We have used colloidal-probe atomic force microscopy to study the viscosity of a class of complex fluids: large molecular weight polymer solutions. The method relies on the use of the deflection of a microcantilever to deduce the viscosity of a liquid confined between a sphere glued on this cantilever and a flat surface holding the liquid sample. The viscosity obtained for a Newtonian fluid is constant and independent of shear rate and separation distance between the sphere and the surface as expected. The large molecular weight polymer solutions, however, exhibit viscoelastic behavior, which is strongly dependent on the gap height. The viscosity measured with this scheme decreases with the decrease of the distance between sphere and surface. This effect is independent of the relative velocity between the flat surface and the probe sphere. These measurements are confirmed by using local viscosity probes in the gap region and are reminiscent of previous results on colloidal suspensions. Depletion of polymers in the gap region due to shear-induced migration is a probable cause for the variation of the viscosity observed here.

\section{ACKNOWLEDGMENTS}

This work was partially supported by the CPER Pôle 4N Nanosciences en Aquitaine (GP-206-action 216/1) with the contribution of the Conseil Régional d'Aquitaine, the FEDER and the Ministry of Education and Research, by the French National Agency (MicRheo Project No. ANR-08-NANO004) and by the Conseil Régional d'Aquitaine (Project No. 20091102001).
[1] H. A. Barnes et al., An Introduction to Rheology (Elsevier, New York, 1989).

[2] F. A. Morrison, Understanding Rheology (Oxford University Press, New York, 2001), p. 560.

[3] R. R. Brau et al., J. Opt. A: Pure Appl. Opt. 9, S103 (2007).
[4] T. G. Mason and D. A. Weitz, Phys. Rev. Lett. 74, 1250 (1995).

[5] D. Wirtz, Annu. Rev. Biophys. 38, 301 (2009).

[6] B. Fabry, G. N. Maksym, J. P. Butler, M. Glogauer, D. Navajas, and J. J. Fredberg, Phys. Rev. Lett. 87, 148102 (2001).

[7] I. Dufour et al., J. Sensors 2012, 719898 (2012). 
[8] G. F. Christopher et al., Lab. Chip. 10, 2749 (2010).

[9] S. Bhattacharya and J. Blawzdziewicz, J. Chem. Phys. 128, 214704 (2008).

[10] G. K. James and J. Y. Waltz, Langmuir 28, 92 (2011).

[11] V. S. J. Craig, C. Neto, and D. R. M. Williams, Phys. Rev. Lett. 87, 054504 (2001).

[12] C. L. Henry and V. S. J. Craig, Phys. Chem. Chem. Phys. 11, 9514 (2009).

[13] E. Bonaccurso, M. Kappl, and H. J. Butt, Phys. Rev. Lett. 88, 076103 (2002)

[14] D. Lasne, A. Maali, Y. Amarouchene, L. Cognet, B. Lounis, and H. Kellay, Phys. Rev. Lett. 100, 214502 (2008).

[15] E. Bonaccurso, Curr. Opin. Colloid Interface Sci. 13, 107 (2008).

[16] C. D. F. Honig and W. A. Ducker, Phys. Rev. Lett. 98, 028305 (2007).
[17] H. Brenner, Chem. Eng. Sci. 16, 242 (1961).

[18] A. J. Goldman et al., Chem. Eng. Sci. 22, 637 (1967).

[19] C. D. F. Honig and W. A. Ducker, J. Phys. Chem. C 111, 16300 (2007).

[20] A. Maali and B. Bhushan, Philos. Trans. R. Soc. A 370, 2304 (2012).

[21] M. D. Graham, Annu. Rev. Fluid Mech. 43, 273 (2011).

[22] Y. Liu, Y. Jun, and V. Steinberg, J. Rheology 53, 1069 (2009).

[23] V. S. J. Craig and C. Neto, Langmuir 17, 6018 (2001).

[24] L. Zhu et al., Langmuir 27, 6701 (2011).

[25] K. L. Linegar et al., Colloid J. 72, 279 (2010).

[26] D. Bonn, F. Ingremeau, Y. Amarouchene, and H. Kellay, Phys. Rev. E 84, 045301(R) (2011).

[27] L. Joly, C. Ybert, and L. Bocquet, Phys. Rev. Lett. 96, 046101 (2006). 